\title{
Improvement and Application of Ant Colony Algorithm Based on Dynamic Candidate List
}

\author{
Hong Zhang ${ }^{1,}$ a, ${ }^{*}$ Zhixin Liu ${ }^{2}$ \\ ${ }^{1}$ School of Economy and Management, Beihang University, China \\ ${ }^{2}$ AVIC Aircraft co. Itd., China \\ azhh58@126.com
}

Keywords: Ant colony algorithm (ACA), partial optimum, dynamic candidate list, entropy.

Abstract. The basic ant colony algorithm converges slowly, is prone to plunge into partial optimum. This paper proposes an improved ant colony algorithm with two highlights. First, dynamic candidate list (DCL) strategy is introduced. In the route construction, candidate routes, whose fitness value surpass some criterion, will be put into DCL. Dynamic candidate strategy is adopted to rapid convergence speed. Second, by using the population's entropy to evaluate the evolution state, the algorithm dynamically adjusts heuristic parameter based on entropy, adapting to different searching stages. The simulation results verify the validity of the improved algorithm.

\section{Introduction}

Ant Colony Algorithm is firstly proposed by Marco Dorigo in his doctoral thesis in $1992^{[1]}$. The algorithm is inspired by the behavior of ants foraging. According to the heuristic idea, the optimal solution to the problem is gradually converging to be solved by the pheromone. Ant colony algorithm has the characteristics of distributed computing, information positive feedback, and has strong robustness, which is easy to be integrated with other optimization algorithms ${ }^{[2]}$.

\section{Ant colony algorithm principle ${ }^{[3]}$}

1) algorithm

Ant colony algorithm is inspired by ant colony foraging behavior. Ants release a substance called pheromone to identify the path, and the following ants determine whether to repeat the path, according to the number of residues in a path. The probability of selecting a path is positively related to the concentration of the residual pheromone of the road, as shown in Figure 1.

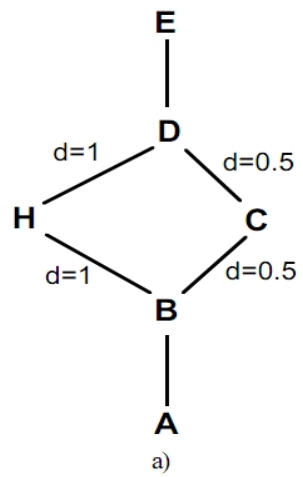

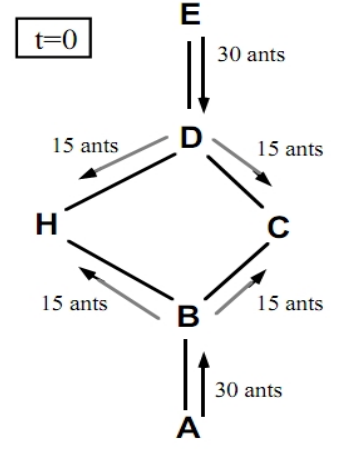

Figure 1: Ant routing process

2) Mathematical model

Assuming $p_{i j}{ }^{k}$ is the state transition probability of the ant $k$ from city $i$ to city $j$, which is not visited city yet. So state transition probability $p_{i j}{ }^{k}$ is: 


$$
p_{i j}^{k}= \begin{cases}\frac{\tau_{i j}^{\alpha}(t) \eta_{i j}^{\beta}(t)}{\sum_{s \in \text { allowed }_{k}} \tau_{i s}^{\alpha}(t) \eta_{i s}^{\beta}(t)} & , j \in \text { allowed }_{k} \\ 0 & , \text { otherwise }\end{cases}
$$

After the first cycle, the ant visited all customer points. When all the ants have completed a traversal, calculate the pheromone increment is calculated, according to the good or bad (fitness function value) of the ant path. The relevant path is updated by the following rules:

$$
\tau_{i j}(t+1)=(1-\rho) \tau_{i j}(t)+\sum_{k=1}^{m} \Delta \tau_{i j}^{k}(t, t+1)
$$

Experiments show that when the scale of the problem is not large (usually 50 100 nodes), the basic ant colony algorithm can achieve good results. But as the complexity of the algorithm is high, it usually takes a long time to search, even Stagnation Behavior may appear ${ }^{[4]}$, i.e. The solutions of all the individuals are in complete agreement, we cannot further search for solution space and conducive to a better solution, after a certain extent search. To solve this problem, many scholars from different angles use different methods to improve the ant colony algorithm, the idea is to build or update these two angles from the path. When the scale of the problem is large, the construction of the candidate list (CL) is an effective method to improve the speed and quality of the algorithm. Dorigo ${ }^{[5]}$ uses the nearest neighbor table (NNL) to build a candidate list, who save the NN for each node and its nearest node for the ants to choose from. Binato $\mathrm{S}$ et al ${ }^{[6]}$ arrange candidate routes in accordance with greedy values in descending order, select the better several routes are added to the candidate list. However, the two algorithms are fixed, we need to carry out many experiments to determine the size of the list size for a specific example. Zhao ${ }^{[7]}$ et al. proposed a new algorithm based on restricted candidate list (RCL), of which candidate table size can be selected randomly, and the shortage of the nearest neighbor candidate list is overcome. But this randomness is likely to lead to the degradation of the algorithm. Paper ${ }^{[8]}$ designed a dynamic candidate list according to the information, but experiments show that when the iteration to a certain stage, with the accumulation of the pheromone leads to the list of the candidates tend to converge to the highest level of information element concentration. So the performance of the algorithm is reduced, and the possibility of local optimum is increased. In this paper, a new dynamic candidate list strategy based on heuristic information is proposed in order to improve the speed of the algorithm.

Meanwhile, the adaptive adjustment of parameters in the process of ant colony algorithm is an important method to improve the performance of the algorithm. For this problem, Cai ${ }^{[9]}$ et al proposed the algorithm of adaptive selection parameters, which dynamically adjusts the route length, based on the degree of information element, expectation and the route length. Xiong et al. ${ }^{[10]}$ proposed a dynamic adaptive algorithm based on greedy strategy. $\mathrm{Li}^{[11]}$ et al. proposed the adaptive adjustment strategy based on the algorithm. The information entropy of the population in the process of Xiao et al.

${ }^{[12]}$ is used to measure the degree of evolution. Thus, the algorithm can adaptively adjust the path selection strategy and the pheromone update strategy. In this paper, we design an adaptive adjustment strategy based on information entropy, which is based on the information entropy of paper ${ }^{[14]}$.

\section{Algorithm improvement}

1) The introduction of dynamic candidate list

In this paper, we choose the greedy function value is greater than the average candidate path value of a certain proportion of the path to join the candidate list (CL). Set time $t$, the ant is located in the node $i$, greedy function is defined as the heuristic information: 


$$
F_{i j}=\eta_{i j}=\frac{1}{d_{i j}}
$$

As shown in Figure 2, for node $i, n-i$ greedy values are obtained. Sort them from big to small: $\left\{\eta_{i}{ }^{1}, \eta_{i}{ }^{2}, \ldots \ldots . \eta_{i}{ }^{n-i}\right\}$, and assume the average value is $\bar{\eta}_{i}$. When greedy values of the candidate paths are: $\mathrm{F}_{l} \geq \gamma \bar{\eta}, \mathrm{CL}$ is added, where $\gamma$ is a coefficient. Only when the candidate path reaches a certain level of quality, it is chosen as the candidate object. In the iterative process, the algorithm adaptively adjusts the number of candidate paths in CL, in order to avoid inferior path to join CL, which leads to a large number of invalid search. The values of the parameter $\gamma$ is determined by experiments, and $0.8 \sim 1.0$ is appropriate value.

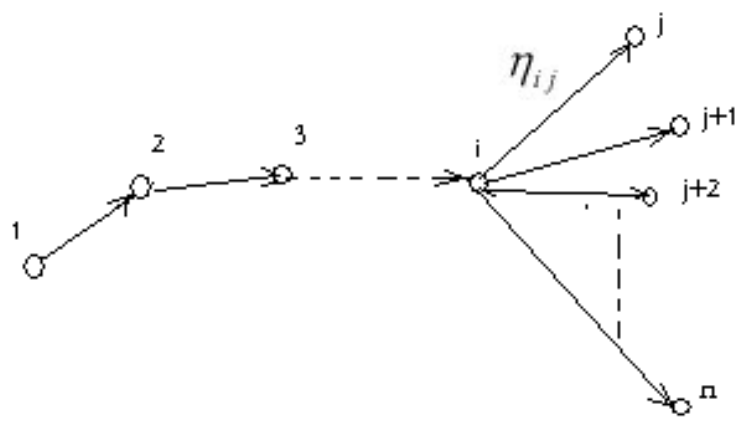

Figure 2: the candidate path diagram

When selecting a path (solution component) to join partial solution, the node $\mathrm{j}$ is re calculated by the average greedy function value $\bar{\eta}_{j}$, and the next line is chosen to construct the new CL, until a complete feasible solution is generated. After the completion of an iterative cycle, the shortest path $T^{\text {is }}$ and (to date) the overall shortest path $T^{g s}$ are obtained. In order to make full use of the existing search knowledge and experience, all the edges of the two paths are set to the top two candidate edges of their corresponding endpoints, and the path selection is preferred. For example, edge (i, j) and edge (i, k) are on $\mathrm{T}^{\mathrm{is}}$ and $\mathrm{T}^{\mathrm{gs}}$ respectively, and the first two elements of the node $i$ candidate list are $\mathrm{j}, \mathrm{k}$.

\section{Algorithm design}

The algorithm of this paper is as follows:

Procedure proposed ACO algorithm

Initialization

Loop

Specify a node for each ant

For $\mathrm{k}=1$ to $\mathrm{m}$ do

Repeat

Calculate candidate list

Select the next node to access

Local pheromone update

Until ant $\mathrm{k}$ has completed a tour $/ *$ Complete a path */

End for

Global pheromone update

Compute entropy value of current pheromone trails $\quad / *$ the calculation of information entropy

$* /$

Modifying the parameter $\beta$.

Until end condition

End 


\section{Summary and conclusion}

In view of the problem that the basic ant colony algorithm has slow convergence speed and easy to fall into local optimum, this paper proposes an improved ant colony algorithm based on dynamic candidate list. The proposed algorithm can only reach a certain level of quality of the candidate path as a candidate, and join the candidate list, narrow the scope of the candidate path, and improve the speed of the algorithm. At the same time, in the calculation of the process according to the change of information entropy of heuristic parameters of dynamic adjustment, to adapt to the characteristics of different phases of ants search in the path selection. The simulation results show that the improved algorithm has a higher quality and better than the max min ant colony algorithm.

\section{Reference}

[1] Dorigo M , Vittorio M, Alberto C. The Ant System: Optimization by a Colony of Cooperating Agents[J].IEEE Transaction Systems, Man, and Cybernetics-Part B,1996,26(1):1-12.

[2] Li Shiyong, ant colony algorithm and its application [M]. Harbin Institute of Technology press, 2004,09:14-16.

[3] Dorigo M, Vittorio M, Alberto C. The Ant System: Optimization by a Colony of Cooperating Agents[J].IEEE Transaction Systems, Man, and Cybernetics-Part B,1996,26(1):1-12

[4] Bi Jun, Fu Mengyin et al., an improved ant colony algorithm for solving the shortest path problem [J]. computer engineering and applications, 2003,39 (3): 107-109.

[5] BAECK T, FUKUDA T , MICHALEWICZ Z. Proceedings of the IEEE International Conference on Evolutionary Computation (ICEC'96) [C].New York: IEEE Press, 1996: 622 -627.

[6] Binato S , de Oliveira G C , de Araujo J L. A greedy randomized adaptive search procedure for transmission expansion planning [J]IEEE Transaction on Power System,2001,16(2):247-253.

[7] Zhao Ling, Liu Sanyang. The response of the restricted candidate list to the TSP problem [J]. Journal of Lanzhou University of Technology, 2006 (04): 83-86.

[8]Dorigo M, Di Caro G. Proceedings of ANT S2002 -From Ant Colonies to Artificial Ants: Third International Works hop on Ant Algorithm Vol2463 of Lecture Notes in Computer Science[ C].Berlin: Springer -Verlag, 2002: 88- 99.

[9] Cai Zhaoquan, Huang Han.Ant colony optimization algorithm based on adaptive weight and volatility parameters[C].Shang-Hai: IEEE Press,2008:75-79.

[10] Xiong Yu. Adaptive ant colony algorithm greedy strategy application in TSP [J]. computer and digital engineering. Based on the 2012 (01): 37-39.

[11] Li Jingyao, Sun Shudong et al. Dual resource constrained job shop scheduling based on adaptive parameter hybrid ant colony algorithm [J]. Journal of Northwestern Polytechnical University. 2011 (01).

[12] Xiao Jing, Li Liangping. Adaptive ant colony algorithm based on information entropy adjustment [J]. computer engineering and design. 2010 (22): 4873-4876.

[13] Xu Guozhi, Gu Jifa, et al. System science [M]. Shanghai: Shanghai Education Press, 2000. 